\title{
Nonequilibrium transitions induced by multiplicative noise
}

\author{
Walter Genovese, ${ }^{1}$ Miguel A. Muñoz, ${ }^{1}$ and J. M. Sancho ${ }^{2}$ \\ ${ }^{1}$ Dipartimento di Fisica, Universitá di Roma “La Sapienza,"' Piazzale Aldo Moro 2, I-00185 Roma, Italy \\ ${ }^{2}$ Department 0407, Institute for Nonlinear Science, University of California, \\ San Diego, 9500 Gilman Drive, La Jolla, California 92093-0407 \\ and Departament d'Estructura i Constituents de la Matéria, Avenida Diagonal 647, 08028 Barcelona, Spain
}

(Received 12 December 1997)

\begin{abstract}
A simple model exhibiting a noise-induced ordering transition (NIOT) and a noise-induced disordering transition (NIDT), in which the noise is purely multiplicative, is presented. Both transitions are found in two dimensions as well as in one dimension. We show analytically and numerically that the critical behavior of these two transitions is described by the so called multiplicative noise (MN) universality class. A computation of the set of critical exponents is presented in both $d=1$ and $d=2$. [S1063-651X(98)51103-8]
\end{abstract}

PACS number(s): 05.40.+j

\section{INTRODUCTION}

It is well established that the effects of noise in stochastic equations can be rather surprising and counterintuitive. The presence of a noisy term can modify in quite an unexpected way the behavior of the corresponding deterministic (noiseless) equation. A well known example of this is the stochastic resonance in which the response to an external periodically oscillating field of a stochastic system is enhanced by increasing its internal noise amplitude [1]. Other examples are the resonant activation [2], noise-induced spatial patterns [3], the noise-induced ordering transition (NIOT) [4-6], and the noise-induced disordering transition (NIDT) [4]. These last two transitions were first analyzed together in an interesting work, to the best of our knowledge, by Van den Broeck, Parrondo, and Toral [4] (VPT hereafter). They considered a Langevin equation with a monostable deterministic term, to which a nontrivial noise is added. Owing to the combined effect of the noise and the spatial coupling two real phase transitions were shown to appear: for small noise amplitudes the system is disordered, while by increasing the noise amplitude the system gets ordered in a symmetrybreaking state (exhibiting therefore a NIOT). If the noise is increased further the ordered state is destroyed (and therefore there is a NIDT). Afterwards, different works on NIOT and NIDT appearing in different models and systems have been proposed in the literature [7-9]. In particular, in [7], a noiseinduced reentrant transition is reported for a time dependent Ginzburg-Landau model with both additive and multiplicative noise. In [8,9] first order transitions are shown to be induced by the presence of nontrivial noises.

An interesting task is that of understanding the nature of this striking phenomena in the simplest possible model: understanding whether it comes from an interplay between additive and multiplicative noises or not, and determining whether the critical behavior of these transitions can be described by any standard universality class. In this direction, the following Langevin equation has been proposed by VPT as a possible simplest model exhibiting these two noiseinduced transitions:

$$
\frac{\partial \phi(x, t)}{\partial t}=F[\phi(x, t)]+D \nabla^{2} \phi+G[\phi(x, t)] \eta(x, t),
$$

with

$$
F[\phi(x, t)]=-\phi\left(1+\phi^{2}\right)^{2}, \quad G[\phi(x, t)]=\left(1+\phi^{2}\right),
$$

where $\phi$ is a field, $\eta$ is a white Gaussian noise with $\langle\eta(x, t)\rangle=0$ and $\left\langle\eta(x, t) \eta\left(x^{\prime}, t^{\prime}\right)\right\rangle=\sigma^{2} \delta\left(x-x^{\prime}\right) \delta\left(t-t^{\prime}\right)$, and the Stratonovich interpretation is considered [10]. Although preliminary simulations seemed to indicate that both the NIOT and the NIDT exhibit exponents compatible with mean-field-like values [4], it has been recently elucidated that both transitions belong in the kinetic Ising model universality class [11]. In particular, very extensive simulations and finite size analysis have been performed [12] showing that all the measured exponents are compatible with their corresponding Ising values. The same conclusion can also be reached by field theoretical analysis [11,13]; and it is also extensible to the model of Ref. [7] with both additive and multiplicative independent noises. In particular, a naive power counting argument permits to conclude that all the terms in Eq. (2) other than the linear, the cubic, and the Laplacian term in the deterministic part, and the constant term in the noise, are irrelevant in the renormalization group sense. In this way, the relevant part of Eq. (2) coincides with the Hohenberg and Halperin model A [14], and consequently Eq. (2) is in the kinetic Ising model universality class. The irrelevant terms, i.e., the $\phi^{5}$ term in $F(\phi)$ and the $\phi^{2}$ term in $G(\phi)$, play a key role in determining the structure of the phase diagram, but do not affect the critical behavior.

In this paper, we face the two previously arisen issues, namely, we propose a candidate with only multiplicative noise exhibiting these two noise transitions, and study their critical behavior under a new perspective. We will conclude that the phenomena is purely multiplicative, and therefore the presence of additive noise [i.e. the constant term in $G(\phi)]$ is not required to generate neither the NIOT nor the NIDT. As a byproduct we show that the conclusion reached in the previous works [12] that both transitions are in the kinetic Ising model universality class is not general. We will show that in the minimal model that we propose, reproduces the phenomena under discussion; the transitions belong into a new recently proposed universality class: the so called $\mathrm{mul}$ tiplicative noise $(M N)$ universality class $[15,16]$. 


\section{THE MODEL}

\section{A. Motivation}

A physically intuitive justification of the NIOT has been recently proposed [12]. It is based on the following observation: the short time evolution of the average value of the field, $\phi$, of a generic Langevin equation of the form (1) with generic forms of the functionals $F$ and $G$, is easily found to be given by

$$
\frac{\partial\langle\phi\rangle}{\partial t}=F(\langle\phi\rangle)+\frac{\sigma^{2}}{2} G(\langle\phi\rangle) G^{\prime}(\langle\phi\rangle)
$$

when $D=0$.

It is shown in $[3,5,7,12]$ that for the models exhibiting a NIOT the previous equation has a linear instability for the homogeneous state $\langle\phi\rangle=0$; this means that the average value of $\phi$ grows at initial times, while it would decrease monotonously towards 0 in absence of the noise term $G(\langle\phi\rangle) G^{\prime}(\langle\phi\rangle)$, i.e., in absence of the multiplicative part of the noise, which is the only one giving a nonvanishing contribution to $G^{\prime}(\phi)$. When the spatial coupling is turned on [i.e., $D \neq 0)$ ], it favors neighbor sites to take similar values of the field, and, consequently, the $\langle\phi\rangle \neq 0$ solution, induced by the multiplicative noise, is stabilized. This is the physical mechanism at the origin of the noise-induced phase transition. At this point we want to stress the fact that is the multiplicative part of the noise the one responsible of the generation of the described short-time instability, and therefore of the NIOT.

On the other hand, the NIDT has a more conventional interpretation, analogous to standard equilibrium transitions: noise (temperature) destroys long range order, thus restoring the symmetry of the homogeneous state.

Inspired by the previous considerations we propose a simpler model to analyze the noise-induced transitions in which the noise is purely multiplicative. In all the previously studied models an additive noise term was also present: it is not essential for the phenomena but it does change the universality class of the two transitions.

\section{B. Definition}

Our model is defined by a Langevin equation (1) with the functionals $F$ and $G$ given by

$$
F[\phi]=-a \phi-\phi^{3}, \quad G[\phi]=\phi\left(1-\alpha \phi^{2}\right)^{1 / 2},
$$

where the Gaussian noise $\eta$ is specified by $\langle\eta(x, t)\rangle=0$ and

$$
\left\langle\eta(x, t) \eta\left(x^{\prime}, t^{\prime}\right)\right\rangle=\sigma^{2} \delta\left(x-x^{\prime}\right) \delta\left(t-t^{\prime}\right),
$$

and $\phi$ is a positive-definite field, and the Stratonovich interpretation is considered.

In this way, we have a very simple deterministic term and a purely multiplicative noise, i.e., with no additive part. By setting the parameter $\alpha$ to zero, we get the NIOT, therefore the more interesting phenomena under study is present in the simple multiplicative noise Langevin equation studied in [15] (that is nothing but our model with $\alpha=0$ ). Nevertheless, that model does not exhibit a NIDT due to the fact that the
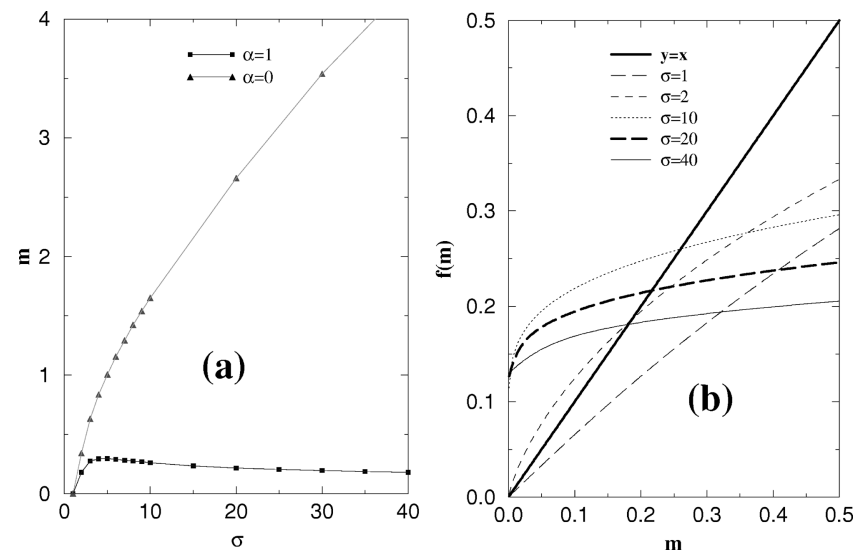

FIG. 1. (a) Mean-field value $m$ of $\langle\phi\rangle$ for different values of $\sigma$, for $\alpha=0$ and $\alpha=1$. (b) Curves $f(m)$ plotted versus $m$ for different values of $\sigma$ and $\alpha=1$. The mean-field solution plotted in (a) is given by the intersection point of the different curves with the straight line, $y=m$ (see text).

order parameter keeps on increasing monotonously with increasing noise amplitude. In order to recover the NIDT a number of possible mechanisms can be invoked. Among them we have chosen to introduce the term in Eq. (5) parametrized by $\alpha$. This "trick" keeps the order parameter from growing indefinitely for large values of $\sigma$. Let us point out that Eq. (4) cannot overpass the upper limit $\phi=\alpha^{-1 / 2}$. Observe that while the multiplicative noise is essential to generate the NIOT, the NIDT is reproduced somehow artificially; the NIDT is more naturally induced by standard additive noise, but it also changes the critical behavior.

\section{Renormalization group results}

Even though we do not present here the details of the calculations, it is a straightforward task to conclude from a naive power counting analysis that this model belongs into the so called MN universality class (defined by our model with $\alpha=0$, see [15]). For that, it has only to be noticed that $\alpha$ is an irrelevant parameter in the renormalization group sense. This conclusion is not altered when introducing diagrammatics corrections. Therefore the critical exponents in all the possible transitions exhibited by this model are expected to be in the MN universality class [15].

\section{Mean-field results}

We have first performed a mean-field analysis of the model. For that we consider a given point $x$ and assume its nearest neighbors to take a constant undetermined value, $m$, the expectation value of $\phi$ is calculated as a function $f$ of $m$, $\langle\phi\rangle=f(m)$, and the equation is closed self-consistently, i.e, $\langle\phi\rangle=m$. In Fig. 1 we observe that, for small values of $\sigma$, $y=f(m)$ does not intersect with the straight line $y=m$, while they do intersect each other for values of $\sigma$ larger than a certain critical value, $\sigma_{c 1}$. The intersection point is very small nearby $\sigma_{c 1}$ and grows while increasing the noise amplitude up to a point in which starts decaying towards zero. It is easy to show from the self-consistency condition that in the infinite noise limit the only solution is $m=0$ when $\alpha$ 


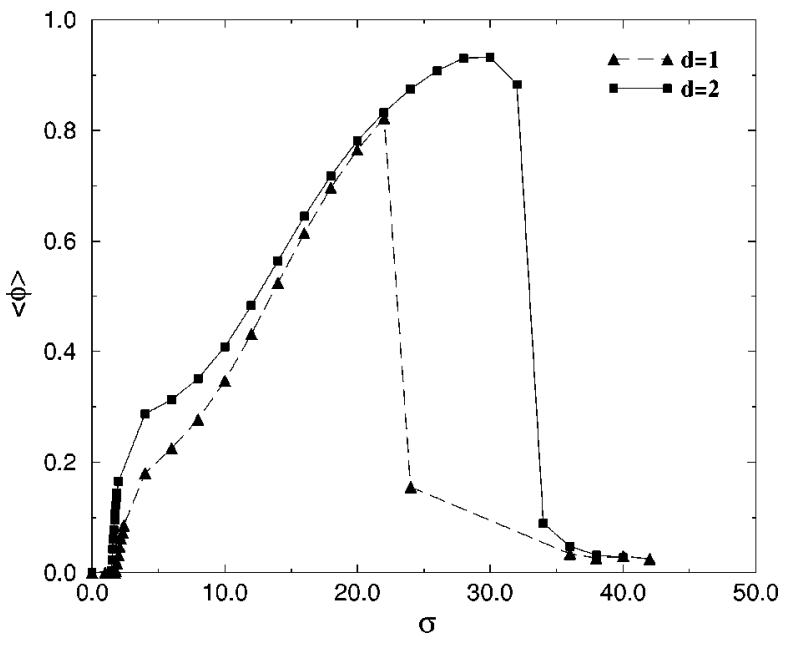

FIG. 2. Stationary expectation value of $\phi$ as a function of the noise amplitude $\sigma$ in one and two dimensions.

$\neq 0$ (this is not the case for $\alpha=0$, for which $m$ diverges). However, the limit $m=0$ is approached in an extremely slow fashion as shown in Fig. 1.

Therefore, in this approximation the model displays a NIOT and a NIDT, and both of them are continuous.

The results for the $\alpha=0$ are also displayed in Fig. 1(a). Observe as it was said previously that the order parameter increases monotonously with $\sigma$ and therefore the model exhibits a NIOT but not the NIDT in mean-field approximation.

\section{E. Numerical results}

We have studied the previous model in both one and two dimensions by integrating numerically the associated Langevin equation. For that we have used a Runge-Kutta type of method (the Heun method $[17,18]$ ). The time step is chosen to be $\delta t=10^{-3}$. Other parameters fixed in the simulations are $a=1, D=0.2$ for the one-dimensional simulations, and $D=1$ in two dimensions. Periodic boundary conditions are employed, and lattice sizes up to $L=1000$ in $d=1$, and $L=40$ in $d=2$. The main results we find are as follows.

(1) We find numerically the two noise-induced transitions in both $d=1$ and $d=2$ for $\alpha=1$ (only the NIOT for $\alpha=0$; in the ordered phase the order parameter grows monotonously with $\sigma$ and therefore there is no NIDT). In Fig. 2 the order parameter, $\langle\phi\rangle$ is plotted as a function of the noise amplitude, $\sigma$, for $d=1$ and $d=2$ (with $\alpha=1$ ). Observe that due to the high values of $\sigma$ the finite size effects are much more pronounced in the NIDT than in the NIOT (as observed previously in [12]), and therefore much larger systems and better statistics are required to determine accurately the critical point and the associated critical behavior of the NIDT.

Let us stress that we find a pair of phase transitions even in $d=1$. This is not expected to be the case in any of the previously studied models due to the fact that in all of them the constant additive part of the noise "cohabits" with the purely multiplicative term, and that changes dramatically the critical behavior, rendering the system Ising-like, and as is well established, systems of that nature cannot get ordered in $d=1$.
(2) We have performed a detailed numerical analysis of the NIOT in one and two dimensions. We have computed the critical exponents defined by

$$
\begin{gathered}
\langle\phi(t \rightarrow \infty)\rangle \sim\left|\sigma-\sigma_{c 1}\right|^{\mu}, \\
\left\langle\phi\left(t, \sigma=\sigma_{c 1}\right)\right\rangle \sim t^{-\theta}, \\
\left\langle\phi^{n}\left(t \rightarrow \infty, \sigma=\sigma_{c 1}\right)\right\rangle \sim\left|a-a_{c 1}\right|^{\beta_{n}} .
\end{gathered}
$$

In order to locate the critical point and determine the exponent $\mu$ in $d=1$ we looked for the value of $\sigma_{c 1}$ for which the best linear fit is obtained in a plot of $\log _{10}(\langle\phi\rangle)$ versus $\log _{10}\left(\sigma-\sigma_{c 1}\right)$. The exponent $\mu$ is given by the slope of such a plot with the optimized value of $\sigma_{c 1}$. We find $\sigma_{c 1}=1.80 \pm 0.02$ with $\mu=0.8 \pm 0.1$ in $d=1$.

Afterwards, we determine $\theta$ from the asymptotic decay of $\langle\phi(t)\rangle$ right at the critical point, and get $\theta=1.0 \pm 0.1$ in $d=1$. Next we fix the noise amplitude to its critical value and perform simulations changing the value of the linear term $a$. By doing that we find $\beta_{1}=1.50 \pm 0.05$ and $\beta_{2}=1.7 \pm 0.2$.

Let us point out that the exponents $\theta, \beta_{1}$, and $\beta_{2}$ are in very good agreement with their corresponding values in the one-dimensional multiplicative noise universality class $[15,19]$, and the predicted scaling relations hold. In particular, we are tempted to conclude from our numerical simulations that $\beta_{1}=\beta_{2}=3 / 2$ and $\theta=1$ in $d=1$ and, in this way, all the exponents in the MN universality class would take rational values. On the other hand, $\mu$ is a new exponent that had not been previously determined for the $\mathrm{MN}$ universality class [20].

In $d=2$ concluding results are much harder to obtain.

We have verified that the behavior of the computed magnitudes at the critical point is compatible with exponents $\mu=1.03 \pm 0.05, \quad \theta=1.75 \pm 0.30, \quad \beta=1.14 \pm 0.05, \quad$ and $\beta_{2}=1.70 \pm 0.10$ [15]. A detailed discussion of this point will be presented elsewhere. To the best of our knowledge this is the first computation of the critical exponents of $\mathrm{MN}$ in $d=2$.

(3) We have also performed a study of the NIDT in $d=1$ and $d=2$. As we have shown, finite-size effects are much more severe in this transition than they are in the NIOT. For that reason, a finite-size-scaling analysis has to be performed in order to elucidate its universality class. In any case, based on both theoretical and preliminary numerical analysis we believe that these transitions also belong into the MN universality class [21]. In particular, the fact that the NIDT is present in $d=1$ excludes the possibility of Ising-like behavior, and relevance arguments strongly support the previous hypothesis.

\section{CONCLUSIONS}

We have introduced a very simple stochastic model exhibiting two noise-induced transitions. We have shown that the NIOT is essentially originated by a purely multiplicative noise, and have found it in $d=1$ (where it has not been previously reported) and $d=2$ (where we measure the MN exponents for the first time). That shows that the multiplica- 
tive noise is capable of originating either a NIOT or a NIDT even in that low dimension in which phase transitions are rare.

We have verified both analytically and numerically that NIOT and NIDT of our model belong into the recently elucidated multiplicative-noise $(\mathrm{MN})$ universality class [15]. The result, obtained in previous works, that the NIOT and the NIDT belong into the kinetic Ising model universality class is not general; that result derives from the fact that an additive noise term is also present in those models, and that type of term, unnecessary as we have shown to generate the phenomena under study, does change the system critical behavior, rendering the universality class model dependent.

\section{ACKNOWLEDGMENTS}

It is a pleasure to acknowledge J. García-Ojalvo for an interesting discussion on the problem studied here, as well as to R. Toral, P. Garrido, and L. Pietronero for useful comments. This work was supported by the European Union through a grant to M.A.M. (Contract No. ERBFMBICT960925), and by Dirección General de Investigación Científica y Técnica, Project No. PB96-0241.
[1] R. Benzi, A. Sutera, and A. Vulpiani, J. Phys. A 14, L453 (1981); K. Weisenfeld and F. Moss, Nature (London) 373, 33 (1995)

[2] C. R. Doering and J. C. Gadoua, Phys. Rev. Lett. 69, 2318 (1992); See also, P. Pechukas and P. Hänggi, ibid. 73, 2772 (1994).

[3] J. García-Ojalvo, A. Hernández-Machado, and J. M. Sancho, Phys. Rev. Lett. 71, 1542 (1993); J. M. Parrondo, C. Van den Broeck, J. Buceta, and F. J. de la Rubia, Physica A 224, 153 (1996).

[4] C. Van den Broeck, J. M. R. Parrondo, and R. Toral, Phys. Rev. Lett. 73, 3395 (1994).

[5] A. Becker and L. Kramer, Phys. Rev. Lett. 73, 955 (1994).

[6] C. Van den Broeck, J. M. R. Parrondo, J. Armero, and A. Hernaández-Machado, Phys. Rev. E 49, 2639 (1994)

[7] J. García-Ojalvo, J. M. R. Parrondo, J. M. Sancho, and C. Van den Broeck, Phys. Rev. E 54, 6918 (1996).

[8] S. Kim, S. H. Park, and C. S. Ryu, Phys. Rev. Lett. 78, 1616 (1997).

[9] R. Müller, K. Lippert, A. Kühnel, and U. Behn, Phys. Rev. E 56, 2658 (1997).

[10] C. W. Gardiner, Handbook of Stochastic Methods (SpringerVerlag, Berlin, 1985).

[11] G. Grinstein, C. Jayaprakash, and Y. He, Phys. Rev. Lett. 55, 2527 (1985).
[12] C. Van den Broeck, J. M. R. Parrondo, R. Toral, and R. Kawai, Phys. Rev. E 55, 4084 (1997).

[13] S. Ramaswamy, R. Pandit, and R. Lahiri, Phys. Rev. Lett. 75, 4786 (1995).

[14] P. C. Hohenberg and B. J. Halperin, Rev. Mod. Phys. 49, 435 (1977).

[15] G. Grinstein, M. A. Muñoz, and Y. Tu, Phys. Rev. Lett. 76, 4376 (1996); Y. Tu, G. Grinstein, and M. A. Muñoz, ibid. 78, 274 (1997).

[16] A. S. Pikovsky and J. Kurths, Phys. Rev. E 49, 898 (1994).

[17] W. H. Press, B. P. Flannery, S. A. Teukolsky, and W. T. Vetterling, Numerical Recipes (Cambridge University Press, Cambridge, 1987).

[18] M. San Miguel and R. Toral, in Stochastic Effects in Physical Systems, edited by E. Tirapegui and W. Zeller (Kluwer Academic, Dordrecht, 1997); cond-mat/9707147.

[19] M. A. Muñoz and T. Hwa, Europhys. Lett. (to be published); cond-mat/9702217.

[20] We have not succeeded so far in relating $\mu$ to other known exponents using scaling arguments.

[21] We cannot rigorously exclude the possibility of the NIDT being discontinuous (first order). 